\title{
Spin-Wave Contribution to the Nuclear Spin-Lattice Relaxation in Triplet Superconductors
}

\section{Citation}

Rostunov, Timofey, Eugene Demler, and Antoine Georges. 2006. "Spin-Wave Contribution to the Nuclear Spin-Lattice Relaxation in Triplet Superconductors." Physical Review Letters 96 (7) (February 22). doi:10.1103/physrevlett.96.077002.

\section{Published Version}

doi:10.1103/PhysRevLett.96.077002

\section{Permanent link}

http://nrs.harvard.edu/urn-3:HUL.InstRepos:27899412

\section{Terms of Use}

This article was downloaded from Harvard University's DASH repository, and is made available under the terms and conditions applicable to Other Posted Material, as set forth at http:// nrs.harvard.edu/urn-3:HUL.InstRepos:dash.current.terms-of-use\#LAA

\section{Share Your Story}

The Harvard community has made this article openly available.

Please share how this access benefits you. Submit a story.

\section{Accessibility}




\title{
Spin-Wave Contribution to the Nuclear Spin-Lattice Relaxation in Triplet Superconductors
}

\author{
Timofey Rostunov, ${ }^{1}$ Eugene Demler, ${ }^{1}$ and Antoine Georges ${ }^{2}$ \\ ${ }^{1}$ Department of Physics, Harvard University, Cambridge, Massachusetts 02138, USA \\ ${ }^{2}$ Centre de Physique Theorique, Ecole Polytechnique, 91128 Palaiseau Cedex, France
}

(Received 21 June 2005; published 22 February 2006)

\begin{abstract}
We discuss collective spin-wave excitations in triplet superconductors with an easy axis anisotropy for the order parameter. Using a microscopic model for interacting electrons, we estimate the frequency of such excitations in Bechgaard salts and ruthenate superconductors to be 1 and $20 \mathrm{GHz}$, respectively. We introduce an effective bosonic model to describe spin-wave excitations and calculate their contribution to the nuclear spin-lattice relaxation rate. We find that, in the experimentally relevant regime of temperatures, this mechanism leads to the power law scaling of $1 / T_{1}$ with temperature. For two- and threedimensional systems, the scaling exponents are 3 and 5 , respectively. We discuss experimental manifestations of the spin-wave mechanism of the nuclear spin-lattice relaxation.
\end{abstract}

DOI: $10.1103 /$ PhysRevLett.96.077002

PACS numbers: 74.25.Nf, 74.70.Kn, 75.30.Ds, 76.60.Es

Nuclear magnetic resonance (NMR) is a powerful tool for analyzing ordered electron states in solids. NMR analysis has been successfully applied to study magnetic insulators (see, e.g., [1], and references therein) as well as several classes of unconventional superconductors, including high $T_{c}$ cuprates [2], heavy fermion materials [3], ruthenates [4], and organic superconductors [5]. In particular, NMR experiments have been useful for analyzing the symmetry of the superconducting order parameter [6] and for clarifying the structure of the phase diagram in systems with competing orders [7].

A common feature of the NMR experiments in certain families of triplet superconductors (TSC) is the power law temperature dependence of the nuclear spin-lattice relaxation rate (NRR). Bechgaard salts [8], ruthenates [4], and heavy fermion materials [9] showed $1 / T_{1} \sim T^{3}$ at low temperatures and for small magnetic fields. Such behavior has usually been interpreted as a signature of nodes in the quasiparticle gap on the Fermi surface. Indeed, point and line nodes should lead to $T^{5}$ and $T^{3}$ scaling of $1 / T_{1}$, respectively. In several cases, however, we have reason to doubt the presence of nodes in the TSC order parameter. For example, in quasi-1D Bechgaard salts, the natural order parameter has different signs on the two sheets of the Fermi surface and no nodal points [10]. Also in ruthenates, the order parameter that is consistent with spontaneous time reversal breaking [11] and the quasi-2D nature of these materials corresponds to a finite quasiparticle gap on the entire Fermi surface [12], although the gap can be strongly inhibited on $\alpha$ and $\beta$ Fermi surfaces relative to the $\gamma$ Fermi surface [13], possibly resulting in node-like temperature dependencies of different physical quantities [14]. In this Letter, we consider a mechanism of the nuclear spin-lattice relaxation that is not due to Bogoliubov quasiparticles but due to collective spin-wave (SW) excitations of the TSC order parameter. We demonstrate that, in the experimentally relevant regime of temperatures, this mechanism also leads to the power law scaling of $1 / T_{1}$ with temperature.

Our starting point is the Moriya relation [15] for the NRR

$$
\frac{1}{T T_{1}}=\frac{2 g_{N}^{2}|A|^{2}}{\left(g_{\text {eff }} \mu_{B}\right)^{2}} \int d^{d} q \frac{\chi_{\perp H}^{\prime \prime}\left(q, \omega_{N}\right)}{\omega_{N}} .
$$

Here $A$ describes the strength of hyperfine interactions between nuclear spins and conduction electrons, $g_{N}$ is a gyromagnetic ratio of the nucleus, $g_{\text {eff }}$ is an effective gyromagnetic ratio of conducting electrons, $\mu_{B}$ is a Bohr magneton, and $\chi_{\perp H}^{\prime \prime}\left(q, \omega_{N}\right)$ is the imaginary part of the transverse (i.e., perpendicular to the magnetic field) electron spin susceptibility taken at the nuclear Larmor frequency $\omega_{N}$. In the case of a perfect spin SU(2) symmetry, linearly dispersing SW excitations exist down to arbitrarily small energies. In real materials, there is always spin anisotropy which gives rise to a finite gap for spin excitations, $\omega_{0}$. Below, we estimate the value of $\omega_{0}$ to be tens of millidegrees Kelvin for Bechgaard salts and hundreds of millidegrees Kelvin for the ruthenates. This is much larger than the nuclear Larmor frequency $\omega_{N}$ but smaller than the typical temperature used in experiments. When $\omega_{0}$ is much larger than $\omega_{N}$, creation and annihilation of individual SWs does not affect $\chi^{\prime \prime}\left(\omega_{N}\right)$. However, there is a contribution due to the scattering of thermally excited SW excitations. Let $\rho(E)$ be the density of states for SW excitations and $n(E)=\left(\exp \left(E / k_{B} T\right)-1\right)^{-1}$ be the Bose distribution function. From the second order perturbation theory, we have $\chi_{z z}^{\prime \prime}\left(\omega_{N}\right) \sim \int \rho(E) \rho\left(E+\omega_{N}\right) \times$ $\left[n(E)-n\left(E+\omega_{N}\right)\right] d E$. The characteristic energy scale in this integral is set by the temperature $T$. Since $T \gg$ $\omega_{0}$, we can assume linear dispersion of SW excitations and take $\rho(E) \sim E^{d-1}$, where $d$ is the number of spatial dimensions. Using $\omega_{N} \ll T$, we have $\chi_{z z}^{\prime \prime}\left(\omega_{N}\right) \sim$ $\omega_{N} \int E^{2 d-2}(-\partial n / \partial E) d E \sim T^{2 d-2}$. Combining this result with the Moriya relation (1), we obtain $1 / T_{1} \sim T^{2 d-1}$. This 
simple analysis does not take into account coherence factors in the expression for $\chi^{\prime \prime}$. Below, we demonstrate that coherence factors do not modify the scaling exponent of the NRR in TSC [16]. This is in contrast to antiferromagnetic (AF) systems that also have linearly spin waves but in which coherence factors contribute an additional $1 / T^{2}$ factor to $1 / T_{1}[1]$.

For a detailed analysis of the NRR, we introduce an effective model that captures the essence of triplet superconductivity and allows us to analyze collective excitations. A simple picture of the TSC state corresponds to binding electrons into Cooper pairs with spin one and momentum zero and Bose condensing such pairs. In an effective bosonic model, one can neglect details of the orbital nature of Cooper pairs and consider them as "elementary" particles. Interactions are important for the correct description of collective excitations; thus, we are led to the Hubbard-type model for spin one bosons [17]

$$
\begin{aligned}
\mathcal{H}= & -t \sum_{\langle i j\rangle \sigma}\left(a_{i \sigma}^{\dagger} a_{j \sigma}+a_{j \sigma}^{\dagger} a_{i \sigma}\right)-\delta r \sum_{i} a_{i 0}^{\dagger} a_{i 0} \\
& +\frac{U_{0}}{2} \sum_{i} n_{i}\left(n_{i}-1\right)+\frac{U_{2}}{2} \sum_{i} \vec{S}_{i}^{2}-\mu \sum_{i} n_{i} .
\end{aligned}
$$

Here $a_{i \sigma}^{\dagger}$ creates a boson on site $i$ with spin $\sigma=\{-1,0,1\}$. Operators $n_{i}$ and $\vec{S}_{i}$ describe the number of atoms and the total spin on site $i: n_{i}=\sum_{\sigma} a_{i \sigma}^{\dagger} a_{i \sigma}, \vec{S}_{i}=\sum_{\sigma, \sigma^{\prime}} a_{i \sigma}^{\dagger} \times$ $\vec{T}_{\sigma \sigma^{\prime}} a_{i \sigma^{\prime}}$, where $\vec{T}_{\sigma \sigma^{\prime}}$ are the usual spin matrices for spin one particles. The first term in the Hamiltonian (2) describes tunneling of Cooper pairs between neighboring lattice sites $i$ and $j$. An important aspect of the model (2) is the presence of two types of interaction terms. The third term in (2) depends only on the number of particles on each site and is the same as for spinless bosons. The fourth term in (2) gives spin dependence to the interaction [without breaking the spin $\mathrm{SU}(2)$ symmetry] and is a novel feature of spinful Cooper pairs. The sign of $U_{2}$ determines the difference between unitary $\left(U_{2}>0\right)$ and nonunitary $\left(U_{2}<\right.$ 0 ) triplet superconductors. In the unitary case, Cooper pairs do not have an expectation value of the spin, and the triplet superconducting order parameter, given by the so-called $d$ vector [see Eq. (8) for the definition of the $\mathbf{d}$ vector], factorizes as $\vec{d}=e^{i \phi} \vec{n}$, where $\vec{n}$ is a real vector. It is generally believed that triplet pairing between fermions leads to unitary Cooper pairs [12]. Thus, from now on we assume that $U_{2}$ is positive. For concreteness, we consider a $d$-dimensional $(d=2,3)$ hypercubic lattice. Our results, however, do not depend on the precise lattice structure. The second term in Eq. (2) introduces easy axis anisotropy for the order parameter by making the condensation of $a_{0}$ to be energetically favorable. A state with finite $\left\langle a_{0}\right\rangle$ corresponds to the unitary state of Cooper pairs with the $\mathbf{d}$ vector pointing along the $z$ axis.

In the mean field approximation, we take $\left\langle a_{0}\right\rangle=\Psi_{0}$, with $\left|\Psi_{0}\right|^{2}=(\mu+z t+\delta r) / U_{0}$ and $z=2 d$ being the coordination number. Without loss of generality, we can take $\Psi_{0}$ to be real. Fluctuations in the phase of $a_{0}$ correspond to the density (Bogoliubov) mode. To find SW excitations, we need to consider $a_{ \pm}$operators. In the Hamiltonian (2), we replace $a_{0}$ by its expectation value, take the terms quadratic in $a_{ \pm}$, and perform the Bogoliubov rotation $a_{i \alpha}=(1 / \sqrt{N}) \sum_{\vec{k}} a_{\vec{k} \alpha} e^{i \vec{k} \vec{r}_{i}}, a_{\vec{k}+}=v_{k} \gamma_{\vec{k}+}+u_{k} \gamma_{-\vec{k}-}^{\dagger}$, $a_{-\vec{k}-}=u_{k} \gamma_{\vec{k}+}^{\dagger}+v_{k} \gamma_{-\vec{k}-}$. Here $N$ is the total number of lattice sites, $u_{k}^{2}+v_{k}^{2}=\left(\xi_{k}+\Delta\right) / E_{k}, 2 u_{k} v_{k}=-\Delta / E_{k}$, $\xi_{k}=-2 t \sum_{n=1}^{d} \cos \left(k_{n} b\right)+U_{0} \Psi_{0}^{2}-\mu$. In these equations, $b$ is the lattice constant and $\Delta=U_{2} \Psi_{0}^{2}$. We obtain the diagonalized spin-wave Hamiltonian $\mathcal{H}_{0}=$ $\sum_{k} E_{k}\left(\gamma_{k+}^{\dagger} \gamma_{k+}+\gamma_{k-}^{\dagger} \gamma_{k-}\right)$, with $E_{k}^{2}=\xi_{k}^{2}+2 \Delta \xi_{k}$. Operators $\gamma_{k \pm}^{\dagger}$ create SW excitations with $S_{z}= \pm 1$. In the long wavelength limit, we find $E_{k}^{2}=\omega_{0}^{2}+v_{s}^{2} k^{2}$, with $\omega_{0}^{2}=$ $\delta r^{2}+2 \Delta \delta r$ and $v_{s}^{2}=2 t U_{2} \Psi_{0}^{2} a^{2}$.

We need to calculate the electron spin susceptibility in the direction perpendicular to the applied magnetic field. Let $\theta$ be the angle between the $z$ axis (i.e., the direction of the $\vec{d}$ vector) and the direction of the magnetic field (see Fig. 1). We have

$$
\chi_{\perp H}=\sin ^{2} \theta \chi_{z z}+\left(1+\cos ^{2} \theta\right) \chi_{x x} .
$$

It is easy to see that $\chi_{x x}$ and $\chi_{z z}$ are given by the correlation function of SWs

$$
\begin{aligned}
\chi_{x x}(q, \omega)= & 2\left(g_{\text {eff }} \mu_{B} \Psi_{0}\right)^{2}\left(u_{q}+v_{q}\right)^{2} \sum_{\alpha \beta} D_{\alpha \beta}^{R}(q, \omega), \\
\chi_{z z}(q, \omega)= & \left(g_{\text {eff }} \mu_{B}\right)^{2} \sum_{\alpha \beta} \int \frac{d^{d} k d \Omega}{(2 \pi)^{d+1}} U_{\alpha \beta}^{2}(k, k+q) \\
& \times D_{\alpha \alpha}^{R}(k+q, \Omega+\omega) D_{\beta \beta}^{A}(k, \Omega),
\end{aligned}
$$

where we introduced the Nambu-Gorkov-type notations $D_{\alpha \beta}^{R, A}(q, \omega)=\int d t e^{i \omega t} \theta( \pm t)\left\langle T \Psi_{q \alpha}(t) \Psi_{q \beta}^{\dagger}(0)\right\rangle$, with $\Psi_{q \alpha}=\left\{\gamma_{q+}, \gamma_{-q-}^{\dagger}\right\}^{T}, \quad$ and $\quad U_{\alpha \beta}\left(k, k^{\prime}\right)=\delta_{\alpha \beta}\left(v_{k} v_{k^{\prime}}-\right.$ $\left.u_{k} u_{k^{\prime}}\right)+\left(1-\delta_{\alpha \beta}\right)\left(u_{k} v_{k^{\prime}}-v_{k} u_{k^{\prime}}\right)$.

Note that there is a qualitative difference in calculating $\chi_{z z}$ and $\chi_{x x}$. A nonuniform magnetic field in the $z$ direction can scatter the existing thermally excited SWs. Thus, we find a finite imaginary part of $\chi_{z z}$ at small frequencies by considering the quadratic Bogoliubov Hamiltonian. We obtain

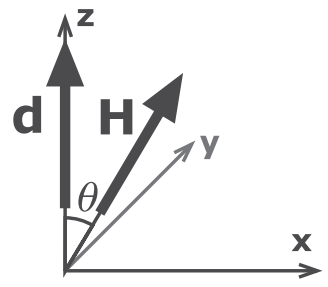

FIG. 1. Orientation of coordinate axes, $\mathbf{H}$, and the $\mathbf{d}$ vector. 


$$
\begin{aligned}
\chi_{z z}^{\prime \prime}\left(\omega_{N}, q\right)= & 2\left(g_{\mathrm{eff}} \mu_{B}\right)^{2} \frac{\omega_{N}}{T} b^{d} \int \frac{d^{d} k}{(2 \pi)^{d}} n\left(E_{k}\right)\left(n\left(E_{k+q}\right)+1\right) \\
& \times \delta\left(E_{k}-E_{k+q}\right) .
\end{aligned}
$$

By contrast, a nonuniform magnetic field in the $x$ direction can only create or annihilate SW excitations. However, energies of these excitations cannot be smaller than $\omega_{0}$. Hence, if we limit ourselves to the quadratic Hamiltonian for SWs, we find that $\chi_{x x}^{\prime \prime}$ is identically zero for frequencies smaller than $\omega_{0}$ at any temperature. To get finite $\chi_{x x}^{\prime \prime}$ at small frequencies, we need to consider interactions between SWs. Taking quartic terms in Eq. (2) and using definitions of SW operators, we obtain the interaction terms between SW excitations. These can be used to calculate self-energies for SW excitations as shown in Fig. 2. We find [18]

$$
\begin{aligned}
\chi_{x x}^{\prime \prime}\left(q, \omega_{N}\right)= & \left(g_{\text {eff }} \mu_{B} \Psi_{0}\right)^{2} \frac{\omega_{N}}{4 \Delta T}\left(U_{0}+3 U_{2}\right)^{2} b^{2 d} \int \frac{d^{d} k_{1}}{(2 \pi)^{d}} \\
& \times \int \frac{d^{d} k_{2}}{(2 \pi)^{d}} \frac{E_{k 1}^{2}+E_{k 2}^{2}+E_{k 3}^{2}}{E_{k 1} E_{k 2} E_{k 3}}\left(n\left(E_{k 1}\right)+1\right) \\
& \times\left(n\left(E_{k 2}\right)+1\right) n\left(E_{k 3}\right) \delta\left(E_{k 1}+E_{k 2}-E_{k 3}\right),
\end{aligned}
$$

where $\vec{k}_{3}=\vec{k}_{1}+\vec{k}_{2}$. We emphasize that Eqs. (5) and (6) apply only in the low frequency limit $\omega_{N} \ll \omega_{0}$ which is relevant for experiments.

Expressions (5) and (6) show that in Eq. (3) contributions to $1 / T_{1}$ due to $\chi_{z z}^{\prime \prime}$ and $\chi_{x x}^{\prime \prime}$ scale as $T^{2 d-1}$ and $T^{3 d-2}$, respectively. For two- and three-dimensional systems, the $\chi_{z z}^{\prime \prime}$ contribution dominates (one can check that this conclusion remains when we include prefactors). At first glance, this result appears surprising. First, the real static susceptibility is finite in the direction perpendicular to the d vector but is zero along it. Second, in the case of full $\mathrm{SO}(3)$ symmetry, creation and annihilation of individual SWs contribute to $\chi_{x x}^{\prime \prime}$ and $\chi_{y y}^{\prime \prime}$ at small frequencies but have no effect on $\chi_{z z}^{\prime \prime}$. A crucial part of our analysis is the existence of a spin gap $\omega_{0}$ which is much larger than the nuclear Larmor frequency $\omega_{N}$. In this case, $\chi^{\prime \prime}\left(\omega_{N}\right)$ does not have contributions due to creation or annihilation of individual SWs. For $\chi_{z z}^{\prime \prime}$, we take thermally excited SWs and scatter them by the magnetic field. For $\chi_{x x}^{\prime \prime}$, we also need thermally excited SWs but, in addition, we must rely on interactions between them. SWs are pseudo Goldstone modes. At low energies, interactions between them are

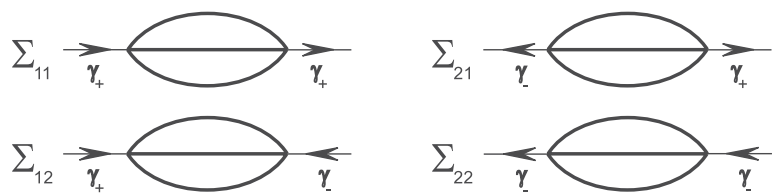

FIG. 2. Second order self-energy diagrams that give rise to scattering of spin waves. For details, see [18]. suppressed. This gives rise to the smallness of $\chi_{x x}^{\prime \prime}$ relative to $\chi_{z z}^{\prime \prime}$.

To summarize, for two- and three-dimensional systems, we find that, as long as $\theta$ is not anomalously small, the SW contribution to the NRR is given by

$$
\frac{1}{T_{1}}=\sin ^{2} \theta|A|^{2} g_{N}^{2} \frac{b^{d}}{v_{s}^{2 d}} \frac{S_{d}^{2}}{4 \pi^{2}}\left|B_{2 d-2}\right| T^{2 d-1} .
$$

Here $S_{d}$ is the surface area of a unit sphere, and $B_{n}$ are Bernoulli numbers, $B_{2}=1 / 6, B_{4}=-1 / 30$. We remind the readers that Eq. (7) applies when $T \gg \omega_{0}$. At low temperatures, $T \ll \omega_{0}$, we expect $1 / T_{1}$ to start decreasing exponentially, reflecting the exponential suppression in the number of thermally excited SW excitations. Equation (7) also predicts that the NRR should be very sensitive to the direction of the magnetic field. We note, however, that this argument is valid only for magnetic fields that are smaller than the so-called spin-flop magnetic field $H_{\text {flop }}$. In magnetic fields larger than $H_{\text {flop }}$, the order parameter $\vec{d}$ will always be perpendicular to the direction of the applied field [18].

It is useful to compare contributions to $1 / T_{1}$ from magnons to the one from quasiparticles. For concreteness, we consider a quasi-2D system with the TSC order parameter $\vec{d}=\Delta_{0} \hat{z} k_{x} / k_{F}$ that has a line of nodes along the $\hat{z}$ axis. The quasiparticle contribution to the NMR relaxation rate in such a state [19] is given by $1 / T_{1 \mathrm{qp}}=$ $\pi^{2}|A|^{2} g_{N}^{2} b^{2} m^{2} T^{3} / 6 \Delta_{0}^{2}$. For comparison, we take Eq. (7) and use the BCS expressions for the velocity of SW excitations (see below). We find $T_{1 \mathrm{mag}} / T_{1 \mathrm{qp}}=\pi^{2} \Delta_{0}^{2} / \epsilon_{F}^{2}$. In a typical superconductor, the value of the quasiparticle gap is much smaller than the electron Fermi energy. Therefore, when we have both gapless quasiparticles and SWs, the quasiparticle contribution will strongly dominate. Only when the quasiparticles are fully gapped out do the magnons provide the dominant contribution to $1 / T_{1}$.

Now we outline the key steps of the analysis that allowed us to estimate the characteristic frequency of SW excitations in TSC. We consider a phenomenological BCStype model for interacting electrons

$$
\begin{aligned}
\mathcal{H} & =\sum_{k \sigma} \epsilon_{k} c_{k \sigma}^{\dagger} c_{k \sigma}-\sum_{a q} V_{a}^{-1}(q) d_{a}^{\dagger}(q) d_{a}(q), \\
d_{a}^{\dagger}(q) & =\frac{1}{2} \sum_{k \alpha \beta} V_{a}(q) f_{k} c_{k+q / 2, \alpha}^{\dagger}\left(i \sigma_{2} \sigma_{a}\right)_{\alpha \beta} c_{-k+q / 2, \beta}^{\dagger} .
\end{aligned}
$$

Here $d_{a}(q)$ are Fourier components of the TSC order parameter in the direction $a(a=x, y, z), f_{k}$ is an orbital wave function [e.g., $f_{k}=\operatorname{sgn}(k)$ [10]], and $c_{k \sigma}^{\dagger}$ are electron creation operators. Parameters $V_{a}$ describe electron interactions in the $p$-wave channel. A homogeneous unitary triplet superconducting state is the ground state of the Hamiltonian (8). Assuming easy axis anisotropy with $V^{z}>$ $V^{x}=V^{y}$, we find $\left\langle d_{z}(q=0)\right\rangle=\Delta_{0}$ and $\left\langle d_{x}\right\rangle=\left\langle d_{y}\right\rangle=0$. To find SW excitations, we consider fluctuations of the TSC order parameter $d_{x}(r, t)=d_{x}^{*}(r, t)$. Integrating out 
fermions gives an effective action for SW excitations [18] $\mathrm{S}_{\mathrm{eff}}\left\{d_{x}\right\}=\int_{\omega q} D_{x}(q, \omega)\left|d_{x}(q, \omega)\right|^{2}$, where $q$ and $\omega$ are the wave vector and the real frequency of the SW, respectively. For the spherically symmetric Fermi surface in $d$ dimensions, we have $D_{x}(\omega, q)=\frac{1}{4} N_{0}\left(\omega_{0}^{2}-\omega^{2}+v_{s}^{2} q^{2}\right)$. Here $N_{0}$ is the density of states at the Fermi energy, $v_{s}^{2}=$ $v_{F}^{2} / d, v_{F}$ is the Fermi velocity, and

$$
\omega_{0}^{2}=4 \Delta_{0}^{2} N_{0}^{-1}\left(V_{x}^{-1}-V_{z}^{-1}\right) .
$$

Zeros of $D_{x}$ correspond to SW excitations. In the case of spin symmetric interactions, SWs are Goldstone modes and are gapless. They have linear dispersion with the velocity $\boldsymbol{v}_{s}$. In the case of the easy axis anisotropy, SWs have a gap $\omega_{0}$.

As a concrete example, we consider the TSC state in Bechgaard salts. We assume that the spin anisotropic part of the interaction in this phase is the same as in the antiferromagnetic state of this material $\Delta \mathcal{H}_{\text {anis }}=$ $\delta J_{z} \sum_{i j\rangle} S_{i}^{z} S_{j}^{z}$. Here $\langle i j\rangle$ corresponds to the nearest neighbor sites and the spin $z$ axis points along the crystallographic $b^{\prime}$ axis. The antiferromagnetic resonance experiments [20] suggest $\delta J_{z}=0.01 \mathrm{~K}$. We can express $\Delta \mathcal{H}_{\text {anis }}$ in the form similar to Eq. (8) with $-\delta V^{z}=\delta V^{x}=\delta V^{y}=$ $\frac{1}{2} \delta J_{z} v_{0}$. Here $v_{0}$ is the volume of the unit cell. Assuming that the anisotropic term is a small correction to the spin symmetric interaction, the total interaction entering Eq. (8) is $V^{a}=V+\delta V^{a}$. The value of $V$ can be estimated from the BCS equation for the transition temperature $T_{c}=$ $1.14 \omega_{\mathrm{BOS}} e^{-1 / N_{0} V}$. Here $\omega_{\mathrm{BOS}}$ is the characteristic frequency of bosons providing electron pairing. Combining all expressions, we find

$$
\frac{\omega_{0}}{\Delta_{0}}=2\left(\delta J_{z} N_{0} v_{0}\right)^{1 / 2} \log \left(\frac{1.14 \omega_{\mathrm{BOS}}}{T_{c}}\right) .
$$

Taking $\delta J_{z}=0.01 \mathrm{~K}, N_{0}=2 \times 10^{33} \mathrm{erg}^{-1} \mathrm{~cm}^{-3}, \omega_{\mathrm{BOS}}=$ $1000 \mathrm{~K}, T_{c}=1.4 \mathrm{~K}, \Delta_{0}=2.5 \mathrm{~K}$, and $v_{0}=360 \AA^{3}$, we obtain $\omega_{0}$ around $1 \mathrm{GHz}$. There is a simple qualitative argument that supports our result that $\omega_{0}$ for Bechgaard salts lies in the gigahertz range. This argument relies on a comparison of SW resonances in the antiferromagnetic and superconducting states of these materials. In quasi-1D systems, microscopic descriptions of the two states are similar, and BCS-type models and our analysis of SW excitations can be used in the antiferromagnetic phase as well. From the discussion above, we expect that the ratio of SW energies in the two phases is approximately proportional to the ratio of AF and TSC transition temperatures, i.e., around 10. In the AF phase, the SW resonance frequency was measured in the tens of gigahertz range [20]. Hence, in the superconducting state, it should be a factor of 10 smaller, which brings us into the gigahertz range.

Similar analysis can be done for the TSC state in $\mathrm{Sr}_{2} \mathrm{RuO}_{4}$. Sigrist and co-workers (see, e.g., Ref. [21]) showed that spin-orbit coupling in these materials leads to a difference in the transition temperature of various order parameters of the order of $2 \%$. From the BCS expression for $T_{c}$, we have $\delta T_{c} / T_{c}=\delta V / N_{0} V^{2}$. Taking $\Delta_{0}=2.6 \mathrm{~K}$, from Eq. (9) we estimate $\omega_{0}$ to be around $20 \mathrm{GHz}$.

In summary, we studied spin-wave excitations in triplet superconductors with the easy axis spin anisotropy. We showed that, in the experimentally relevant regime of temperatures, $1 / T_{1}$ has a power law scaling with temperature, including the $T^{3}$ dependence for two-dimensional systems. We showed that the spin-wave mechanism predicts a dramatic decrease of $1 / T_{1}$ for temperatures lower than the energy of spin-wave excitations and leads to dependence of $1 / T_{1}$ on the direction of the applied magnetic field.

We are grateful for useful discussions with E. Altman, D. Podolsky, and A. Polkovnikov. This work was supported by NSF Grant No. DMR-0132874, the Sloan Foundation, and KITP UCSB.

[1] D. Beeman and P. Pincus, Phys. Rev. 166, 359 (1968).

[2] A. Sakai et al., Physica (Amsterdam) 388C-389C, 251 (2003); P. M. Singer et al., Physica (Amsterdam) 388C389C, 209 (2003); C. P. Slichter et al., J. Phys. Chem. Solids 54, 1439 (1993); V. F. Mitrovic et al., Phys. Rev. B 67, 220503(R) (2003).

[3] H. Tou, K. Ishida, and Y. Kitaoka, J. Phys. Soc. Jpn. 74, 1245 (2005).

[4] K. Ishida et al., Phys. Rev. Lett. 84, 5387 (2000).

[5] S. Lefebvre et al., Phys. Rev. Lett. 85, 5420 (2000); C. Bourbonnais et al., Phys. Rev. B 33, 7608 (1986); Phys. Rev. Lett. 62, 1532 (1989).

[6] I. J. Lee et al., Phys. Rev. Lett. 88, 017004 (2002); Y. Kitaoka et al., Physica (Amsterdam) 281B-282B, 878 (2000).

[7] I. J. Lee et al., Phys. Rev. Lett. 94, 197001 (2005).

[8] I. J. Lee et al., Phys. Rev. B 68, 092510 (2003); M. Takigawa et al., in Novel Superconductivity, edited by S. A. Wolf and V.Z. Kresin (Plenum, New York, 1987).

[9] Y. Kohori et al., J. Phys. Soc. Jpn. 57, 395 (1988).

[10] M. Dressel, Physica (Amsterdam) 317C, 89 (1999).

[11] G. M. Luke et al., Nature (London) 394, 558 (1998); J. A. Duffy et al., Phys. Rev. Lett. 85, 5412 (2000).

[12] A. Mackenzie and Y. Maeno, Rev. Mod. Phys. 75, 657 (2003).

[13] T. Nomura and K. Yamada, J. Phys. Soc. Jpn. 71, 404 (2002).

[14] K. Deguchi et al., Phys. Rev. Lett. 92, 047002 (2004); C. Lupien et al., Phys. Rev. Lett. 86, 5986 (2001).

[15] T. Moriya, J. Phys. Soc. Jpn. 18, 516 (1963).

[16] Note that here we discuss coherence factors for effective bosonic models which are different from the usual electron coherence factors in the BCS-type models.

[17] E. Demler and F. Zhou, Phys. Rev. Lett. 88, 163001 (2002).

[18] T. Rostunov, E. Altman, and E. Demler (unpublished).

[19] M. Sigrist and K. Ueda, Rev. Mod. Phys. 63, 239 (1991).

[20] J. B. Torrance et al., Phys. Rev. Lett. 49, 881 (1982); M. Dumm et al., Phys. Rev. B 61, 511 (2000); 62, 6512 (2000).

[21] K. K. Ng and M. Sigrist, Europhys. Lett. 49, 473 (2000). 